\section{Precautions in ophthalmic practice in a hospital with a major acute SARS outbreak: an experience from Hong Kong}

W-M Chan ${ }^{1,2}$, DTL Liu ${ }^{1}$, PKS Chan ${ }^{3}$, KKL Chong ${ }^{1}$, KSC Yuen ${ }^{1}$, TYH Chiu ${ }^{1}$, BSM Tam¹, JSK Ng ${ }^{1,2}$ and DSC Lam ${ }^{1,2}$

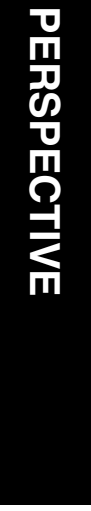

${ }^{1}$ Department of

Ophthalmology and Visual Sciences, Prince of Wales Hospital, The Chinese University of Hong Kong, Hong Kong, People's

Republic of China

A (H5N1) outbreak; infection control measures; ophthalmic practice precautions; Hong Kong

\section{Introduction}

Severe acute respiratory syndrome (SARS) is the 'modern plague' of the new era for its infectivity, novelty, casualty, and high fatality. ${ }^{1,2}$ Hong Kong, Guangdong Province, some other provinces of China, Singapore, Taiwan,

Vietnam, Canada, and the whole world are now under unprecedented threats from this deadly infectious disease. ${ }^{1-8}$ The culprit virulent agent is believed to be a novel Coronavirus strain (SARS-CoV) that is not closely related to any of the previously characterized coronavirus, according to the World Health Organization (WHO) and local infection control experts., ${ }^{4,5}$ The awful impact of this disease can be reflected by the alarming health-care statistics recorded since the massive outbreak in Hong Kong on March 12, 2003. The accumulative number of SARS infection by October 15, 2003 was 1755 with a total number of deaths of 299 and among them eight of the deceased were doctors, nurses, and health-care assistants. ${ }^{7}$

SARS has unveiled many virgin health-care issues that have not caught the attention of the general public in the past and the whole healthcare system in Hong Kong has been significantly percolated and bombarded by SARS in terms of changes in daily practices and everyday routines. Ophthalmology is no
${ }^{2}$ Department of Ophthalmology and Visual Sciences, Hong Kong Eye Hospital, The Chinese University of Hong Kong, Hong Kong, People's Republic of China

${ }^{3}$ Department of Microbiology, The Chinese University of Hong Kong, Prince of Wales Hospital, Shatin, New Territories, Hong Kong, People's Republic of China

Correspondence: DSC Lam, Department of

Ophthalmology and Visual Sciences, The Chinese University of Hong Kong, 3/F., Hong Kong Eye Hospital, 147 K Argyle Kong, People's Republic of China

Tel: + 85227623157 ; Fax: + 85227159490

E-mail: dennislam@ cuhk.edu.hk

Received: 24 May 2004 Accepted: 22 February 2005 Published online: 29 April 2005

Financial and proprietary interest: Nil Financial support: Nil Street, Kowloon, Hong 
exception. The Ophthalmologist, as one of the frontline health-care providers, has to inevitably face these new challenges and the ever-changing expectation upon our profession from patients and the community. Several clinical problems are particularly pertaining to the ophthalmic practice. Firstly, what we can be certain right now is that this novel Coronavirus-associated atypical pneumonia is extremely infectious as reflected by the high prevalence of infected health-care workers. By October 15, 2003, 386 of 1755 patients (21.9\%) were health-care workers of hospitals/clinics or medical students. Most of them had a history of close contact with known or finally known SARS patients. ${ }^{7}$ It is still unclear whether ophthalmologists are at increased risk of infection in examining a proven or suspected SARS patient with short working distance, especially in performing direct ophthalmoscopy and slit-lamp examination.

Secondly, the route of transmission is still enigmatic and the relative infective roles of different body fluids are still under active study. ${ }^{1,2}$ Droplets and fomites are established main routes of transmission. Stool and urine have also been implicated as the possible media in harboring the infective agent. ${ }^{1-3}$ Tears and eye discharges are body secretions that may be equally or potentially more hazardous to health-care personnel, particularly ophthalmologists. In fact, tears have been reported by WHO to be one of the body fluids conveying the novel SARS-CoV, although the infectivity or clinical significance is still not known for the time being. ${ }^{8}$ The use of different instruments, which are in direct contact with patients' conjunctival mucosal surface may serve as possible routes and sources for disease transmission. The ultimate infectivity of the tears secretion and ocular discharge from SARS patients may bring impacts on not only the daily ophthalmic practice but also the universal infection control measures practiced by general public and health-care workers.

Being the Ophthalmology Department of the only hospital in the world that has just gone through the largest outbreak of SARS, we would like to share our strategy, measures and experience of preventing SARS infection.

\section{Scenario report of a suspected case during the hospital outbreak}

In early March 2003, shortly after the SARS outbreak within a medical ward in the Prince of Wales Hospital, Hong Kong, a 39-year-old lupus woman admitted under eye ward for elective operation of wound revision of an enucleated eye developed a high fever of $39.2^{\circ} \mathrm{C}$ on postoperative day 1 after an uneventful operation. Supplementary oxygen was required to alleviate the shortness of breath and oxygen desaturation. Chest radiography showed bilateral lung parenchymal infiltrates without consolidation. Respiratory physician recognized her condition as a probable case of SARS that required Intensive Care Unit admission with ventilatory support. At 3 days prior to the present admission, she had been seen by two of our colleagues in the outpatient consultation room. Immediate notification of the Infection Control Unit of the hospital was performed. The SARS Command Centre of the Department of Health of Hong Kong and the Hospital Authority consisting of infection disease clinicians, microbiologists, and epidemiologists were notified as well. In accordance with the infection surveillance protocol, the clinic doctors and nurses, who had a close contact with the patients, were required to have compulsory blood check for cell counts and chest radiography despite the fact that they did not exhibit any symptoms on initial assessment. The investigations were normal but they still have to be put on home quarantine for 7 days. Subsequently, the policy was further geared up to 10 days home quarantine for those health-care workers having unprotected contacts of similar setting. The diagnosis of the patient was finally revised and confirmed to be lupus pneumonitis with systemic flare-up. She succumbed on day 11 after admission. With such an experience, we have consolidated both the infection control and containment measures with respect to in-patient and outpatient ophthalmic services.

\section{Preferred ophthalmic practice for infection surveillance and control in epidemic areas or hospitals with SARS outbreak}

The level of infection control and precautions could be specifically targeted according to the level of regional epidemic, different clinical settings, and scenarios as follows.

\section{Outpatient care}

The eye clinic is one of the most crowded areas, with many patients. Suboptimal infection control strategies in the clinic may inadvertently help the spread of SARS infection and jeopardize the whole community infection containment policy. We have used modified WHO cases classification schemes in handling our eye patients in the clinic. Three categories of patients are recognized based on a triage system run by experienced nurses. They are the general, suspect, and probable categories. ${ }^{3-5}$

\section{General categories}

In attending all ophthalmic outpatients, irrespective of their SARS status, staff are encouraged to follow the 
universal precaution measures consisting of wearing surgical/N95 respirator mask, wearing gloves in handling blood, body fluids, secreta and excreta, wearing water-repellant or water-resistant gowns and goggles (visor) during splashes or sprays generating procedures such as measuring noncontact tonometry (NCT) (Table 1). The most important of all is frequent hand washing, preferably with the use of chlorhexidine alcoholic handrub (0.5-1.0\% chlorhexidine in $80 \%$ ethyl alcohol, or a similar commercial product, such as $\mathrm{Hexol}^{\circledR}$ ) in the prevention of crossinfection. The gloves, if used for high-risk procedures, must be changed and hand hygiene must be performed in between each contact with different patients. Staff are strongly advised not to touch their face-shield, eye protective wear, mask, head, and neck region before a thorough and complete hand washing procedure. Visitors are restricted in the hospital or clinic complexes unless under exceptional circumstances such as the escort of demented or visually impaired patients or parents of small children. ${ }^{3}$

\section{Suspect and probable categories}

For known cases of SARS inpatients, the outpatient attendance is deemed contraindicated because of significant risk of crossinfection. Any ophthalmic consultation should be completed within the quarantine ward instead. If an outpatient presented with overt SARS symptoms and signs, immediate attention from the respiratory physicians and infection control officers should be summoned. The ophthalmic appointment should be deferred indefinitely until the status of infection is ascertained.

For patients presenting with fever, contact history of SARS patients within 10 days, or recovered SARS patients shortly discharged from hospital, we have adopted a special outpatient follow-up strategy based on initial epidemiological and microbiological evidences that the main mode of transmission of SARS is by droplets, direct contact with patient's secretions, and subsequent inoculation into mucous membranes. ${ }^{3}$ Infection control measures accentuating droplet precautions, with the application of barrier apparels and environmental cleaning as well as universal precautions, being the pivot of success in containing the infection, are of paramount importance. The personal protective equipment (PPE) with disposable caps, N95 respirator, goggles, face-shields, gloves, top and pants, and long protective gowns with high neck and long cuffed sleeves should be worn all the time when attending to these patients (Table 1). Under circumstances such as between cases and immediately following a high-risk procedure, all health-care workers have to abide by the decontamination process consisting of removal of all potentially contaminated protective wear in proper sequence and putting on clean protective wear. Once again, hand washing is strongly advised after removal of gloves and before attending another patient in order to minimize risk of crossinfection.

It is very advisable that the suspect category patients be seen during the last time slot at which most of the patients of the general category have left the clinic already. A specifically designated consultation room with separate set of examining instruments and independent ventilatory system is highly desirable. The ventilatory system is preferably of exhaust fan type with filter, generating a negative room pressure with lamellar downward airflow. During the ophthalmic examination, patients themselves are required to put on the N95 respirator mask as well. Self-adhesive surgical plastic sheets that are commonly used during cataract surgery

Table 1 Personal protective equipment (PPE) in outpatient ophthalmic care

\begin{tabular}{|c|c|c|}
\hline & General category (universal precaution measures) & Suspect and probable categories of SARS (special measures) \\
\hline Disposable cap & Standard & Standard \\
\hline \multirow[t]{2}{*}{ Eye protection } & $\begin{array}{l}\text { Visor or goggle - advisable when in direct contact } \\
\text { with patient }\end{array}$ & Face shield -standard in contact with patient \\
\hline & Face-shield — for high-risk procedures & $\begin{array}{l}\text { Goggle in addition to face shield - for high-risk } \\
\text { procedure }\end{array}$ \\
\hline Mask & $\begin{array}{l}\text { Surgical mask - for routine setting } \\
\text { N95 respirator — for high-risk procedure }\end{array}$ & N95 respirator \\
\hline Gown & Water repellent gown or water-resistant gowns & $\begin{array}{l}\text { Water repellent gown or Barrier-Man }{ }^{\mathbb{R}} \text { or similar } \\
\text { protective attire }\end{array}$ \\
\hline Gloves & $\begin{array}{l}\text { Hand washing or use alcohol-rub on hands in- } \\
\text { between cases } \\
\text { Wear glove in high-risk procedure }\end{array}$ & $\begin{array}{l}\text { Wear gloves. Discard gloves, wash or alcohol-rub the } \\
\text { hands and then put on new gloves in-between case }\end{array}$ \\
\hline
\end{tabular}

General categories: for all patients attending the ophthalmic outpatients in which the SARS status is not certain.

Suspect and probable categories: for any known or suspect cases of SARS from the history, symptoms or signs.

High-risk procedures in ophthalmology: include those with short-distance contact, prolonged contacted, and involving splashes or sprays generating actions. Direct ophthalmoscopic examination, irrigation and probing, nasal endoscopic examination, laser photocoagulation, measurement by noncontact tonometry, ophthalmic surgeries are some examples. 
can be applied directly but lightly over the face and the nostril areas, leaving behind the eyes for examination. This innovative infection control measure specific for eye examination can help preventing any splashing of droplets from inadvertent coughing or deep breathing.

In SARS, SARS-CoV could be transmitted through the contaminated hands and instruments in addition to droplets. The strong survival ability of SARS-CoV over surfaces of commonly used materials in domestic and working environments have been quantitatively proved by in vitro models. ${ }^{9}$ Therefore, the disinfection of the ophthalmic instruments as well as the environmental surfaces in frequent contact with patients are deemed as one of the key infection containment measures. Susceptibility of human coronaviruses 229E and OC43 to chemical disinfection has been demonstrated by biological viral titration assay, ${ }^{10,11}$ whereas similar in vitro data concerning the efficacy of different hospital disinfectant products (US Environmental Protection Agency (EPA) registered disinfectants) against this novel coronavirus (SARS-CoV) is currently lacking. ${ }^{12}$

Nevertheless, we can still make use of the available chemical germicides that could inactivate related viruses with similar physical and biochemical properties as the possible SARS agents. The adopted disinfection protocols in our ophthalmic practice are in line with the recommendation by the Centers for Disease Control \& Prevention ${ }^{13}$ and based on the scientific evidences provided by APIC guidelines. ${ }^{14}$

Ophthalmic instruments especially those in direct contact with patients' mucosal membranes like the Goldmann applanation prism tip and Goldmann contact lens require intermediate level of disinfection. ${ }^{14}$ They should be immediately and separately disinfected with $2 \%$ alkaline glutaraldehyde $\left(\right.$ Cidex $\left.^{\mathbb{R}}\right)$ for $20 \mathrm{~min}$ at room temperature or $6 \%$ hydrogen peroxide after use. ${ }^{14}$ Frequent (at least daily) disinfection of the apparatus (slit lamp, binocular indirect ophthalmoscope, etc) with hypochlorite $(\mathrm{NaOCl})$ solution $(1000 \mathrm{ppm}, 1$ in 50 dilution of $5.25 \%$ household bleach, for $2 \mathrm{~min}$ ) is mandatory. ${ }^{14}$ The examination table and slit lamp will be cleaned and disinfected thoroughly before another patient is seen in that environment. Surfaces that cannot be easily cleaned, such as keyboards of desktop computers, should be covered within plastic sheeting, wiped between cases, and changed at the end of the day. If the plastic wraps are grossly soiled with patient's tears or ocular secretion, they should be immediately removed and replaced. Daily cleaning and disinfection of the environmental surfaces (eg, door knobs, phone, lavatory facilities) that have been frequently touched by patients and health-care workers are specifically targeted, in accordance with the recommendations by the CDC. ${ }^{12,13}$

Unless it is otherwise indicated, the consultation waiting time and overall consultation time ought to be maintained at a safe minimum. After all, the outpatient attendance should be judiciously kept at a low level so as to curtail the risk of crossinfection.

\section{In-patient care}

The different wards in hospital have been stratified into 'high risk' and 'all other areas'. High-risk areas refer to intensive care unit (ICU), SARS ward, Infection triage or Cohort fever ward, Accident and Emergency Department, Admission or Observation wards.

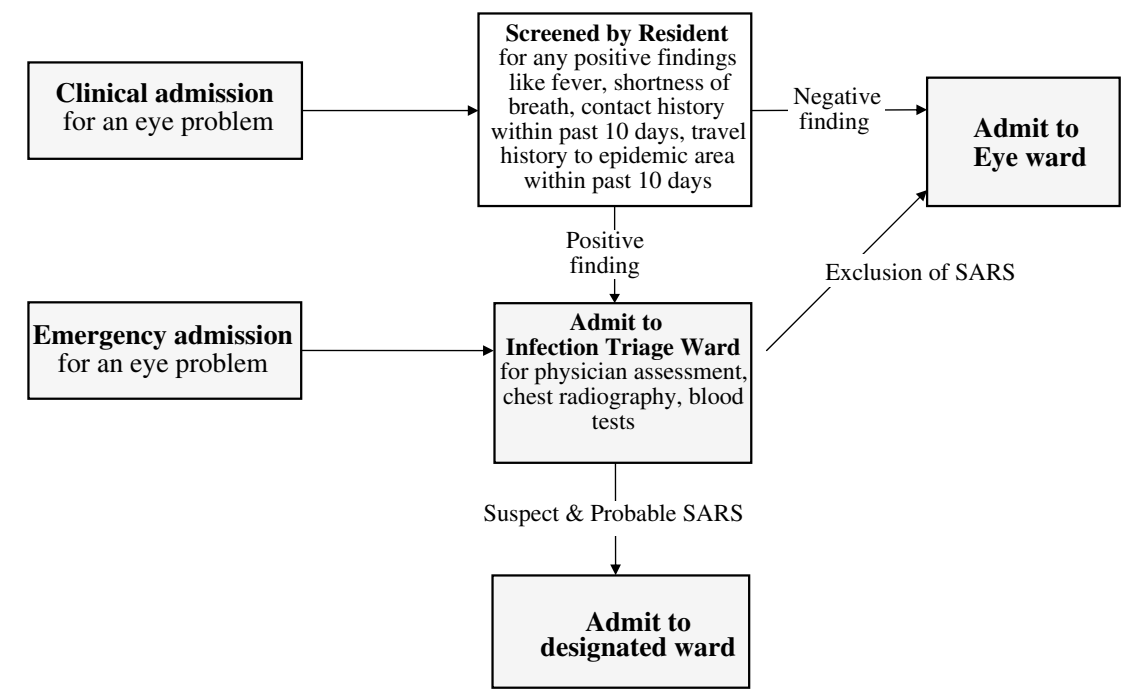

Figure 1 Flow chart for any clinical or emergency admission to eye ward. 


\section{Ophthalmic ward}

The eye ward belongs to the category of 'all other areas'. All patients admitted to the eye ward will be screened for symptoms and signs of SARS by the house eye surgeon or resident (Figure 1). Any positive findings like fever, recent contact history or travel history to epidemic area will call for an admission to a centralized observation ward or infection triage ward instead. Patients will be observed for at least $24 \mathrm{~h}$ and will be examined by the respiratory physician in addition to radiological investigation of the chest (chest radiography or computerized tomography thorax). Suspect or probable cases will be transferred to another infection quarantine ward for further observation and management. Once again, universal infection control and containment measures are practiced at all times. The PPE with the same level as 'suspect \& probable categories of SARS' in OPD should be adopted (Table 1).

Ophthalmic consultation from other wards or specialties Every consultation is screened by an ophthalmologist and the degree of urgency of the consultation is determined. For nonurgent consultations, appropriate outpatient appointment will be granted accordingly, provided patients can be discharged from hospital for at least 10 days before the appointment. In case of ophthalmic emergency or semiurgent cases (acute primary or secondary glaucoma, penetrating ocular trauma, alkali chemical injuries and the like), patients will be seen accordingly in their original wards. Transfer of in-patients to the eye outpatient department or to the eye ward for the purpose of examination is regarded as absolutely contraindicated for the sake of preventing cross infection. We have arranged our eye colleagues to examine the patients within their original wards by a portable slit lamp, Tonopen, and binocular indirect ophthalmoscope. Proper and thorough disinfection of the instruments will be carried out by eye ward nursing staff after each consultation. In dealing with suspect or probable SARS patients in other wards, ophthalmologists should maintain a high index of caution with the full standard barrier apparels or protective attire in carrying out short-range procedures or examinations.

\section{Ophthalmic operation and intervention}

Nonurgent ophthalmic operations like cataract operations, eyelid surgeries and squint extraocular muscle surgeries, and nonurgent ophthalmic interventions performed in outpatients such as barrier laser, pan-retinal photocoagulation, YAG:Nd laser capsulotomy, probing and syringing, incision and curettage will be performed selectively and proper precautions ought to be taken.
Ocular emergencies such as macula-on rheugmatogenous retinal detachment, ruptured eyeball and intraocular foreign body will be dealt with by arranging an emergency operation. The operating theater is regarded as a high-risk area for SARS infection and universal precaution measures with barrier apparels (N95 and surgical mask, goggles, plastic apron, operating theater gown, and gloves) will be strictly practiced.

\section{Lessons to learn}

Given the facts that the SARS-CoV is highly infectious, and the observation that none of the staff and patients has been infected within the Department of Ophthalmology, in both in-patient and outpatient areas throughout this massive outbreak, it is an encouraging and vindicating efficacy of the precautions adopted. We, as ophthalmologists, had also safely performed several clinical studies involving direct patient contacts, close examinations and performance procedures of such as conjunctival scrapping, during the SARS attack. ${ }^{15,16}$

Universal precautions during the SARS period are the basic measures. In a case-control study among 254 Hong Kong health-care workers with documented exposure to SARS patients, none of the 69 staff reporting use of four infection control measures, namely mask, gloves, gowns, and hand washing, was infected. ${ }^{17}$ On the contrary, at least one of the four measures had been omitted $(P=0.0224)$ among all 13 infected staff. In a comparison between infected and noninfected cohorts, health-care personnel practicing hand washing $(P=0.011)$ and personal protective equipments (wearing masks and gowns) were significantly associated with fewer infections. These data provide, at least to a certain extent, scientific rationale for our infection control precautions.

Ophthalmologists will inevitably get into contact with the tears and sometimes conjunctival mucosa of patients and unlike other body fluid or secreta, ${ }^{1,2}$ the infectivity of tears from SARS patients remains elusive in spite of preliminary studies. ${ }^{15,18}$ In the face of these uncertain infective risks and inadvertent close contact with patients, ophthalmologists may need to maintain extra vigilance with regard to infection control. In real life, the ophthalmic practices in the midst of the SARS outbreak have been changed. The ophthalmologists in Hong Kong have abandoned the direct ophthalmoscopic examination in view of its short working distance. In ultrahigh risk patients proven to have SARS, safer and easily accessible investigative tools like fundus photography might be an alternative to conventional fundus examination.

The knowledge obtained from the SARS outbreak in our hospital has been remarkable. It demonstrates the significance of infection control in clinical practice. Strict 
infection control and containment at the appropriate level according to the infectivity, route of transmission, morbidity, and fatality caused by the suspected organism is important in preventing crossinfection.

\section{Conclusions}

SARS infection has swept through the continents and caused hundreds and thousands of mortality and morbidity. The cumulative numbers of probable SARS worldwide by Sept 26, 2003 was 8098, in which 1707 (21\%) were health-care workers. A total of 774 patients died from the disease with a fatality ratio of $9.6 \% .{ }^{19}$ It was first recognized as a global threat in mid-March 2003, but was successfully contained in less than 4 months with international cooperation. On 5 July 2003, WHO reported that the last human chain of transmission of SARS had been broken. ${ }^{20}$

The epidemic, however, is not yet over. Firstly, sporadic confirmed and probable SARS-CoV infection have been reported and it is important to note that the source of infection in the most recent case is still unknown. ${ }^{21}$ Secondly, even with the aid of molecular epidemiological techniques such as genetic fingerprinting, the natural reservoir, host, vector and media of transmission of SARS-CoV, is still enigmatic to mankind. ${ }^{22,23}$ Owing to lack of a clear understanding of the epidemiological characteristics, a complete eradication of the SARS-CoV is remote and the world anticipates ongoing new cases. Thirdly, mutation analysis has demonstrated continuous evolutional change of the genetic constitutions of SARS-CoV, which may encourage emergence of more virulent strains in a community without immunity against this pathogen. ${ }^{24}$ With an interference of the human-animal ecology, more new virulent species are imminent, SARS-CoV infection is an example, and avian-flu is another. Countries that were not involved during the heat of the outbreak have recently stepped up this national SARS preventive measures. ${ }^{25}$ In the postoutbreak period, all health-care workers, including ophthalmologists, should remain vigilant for the recurrence of SARS and always incorporate risk-based infection control measures in care provision.

SARS is one of the many viruses against which personnel will need protection in an ophthalmic setting. The experiences we have learnt also apply to other highly infectious disease, which can be spread by means of droplets. ${ }^{26}$ Avian influenza A (H5N1) is an example. ${ }^{27}$ It is a viral infection that can affect humans and cause serious disease and death. ${ }^{27,28}$ There is still no solid evidence of human-to-human transmission in most of the outbreaks involving human cases. ${ }^{27}$ However, a recent clustering of cases in a family, probably resulting from person-to-person transmission during unprotected exposure to the critically ill index patient was recorded. ${ }^{28}$ These findings indicate that inefficient transmission is possible and reinforce the importance of infection control precautions among health-care workers. ${ }^{29}$ If $\mathrm{H} 5$ viruses do persist, they will likely continue to evolve, or reassort with existing human influenza viruses, to become more virulent and more easily transmitted subtypes from person-to-person. ${ }^{26,30}$ We must be well prepared for any $\mathrm{H} 5 \mathrm{~N} 1$ outbreaks as it is difficult to eradicate the existing virus in nature with the potential reservoirs in wild birds. ${ }^{30}$ Strict adoption of the regularly revised infection control guidelines in accordance to the updated clinical, epidemiological, and laboratory information is the only way to prevent further large-scale hospital outbreak or crossinfection.

\section{References}

1 Lee N, Hui D, Wu A, Chan P, Cameron P, Joynt GM et al. A major outbreak of severe acute respiratory syndrome in Hong Kong. N Engl J Med 2003; 348: 1986-1994.

2 Tsang KW, Ho PL, Ooi GC, Yee WK, Wang T, Chan-Yeung M et al. A cluster of cases of severe acute respiratory syndrome in Hong Kong. N Engl J Med 2003; 348: 1977-1985.

3 Hospital Authority of Hong Kong. Hospital Authority guideline on infection control of Severe Acute Respiratory Syndrome (SARS). Aug 1, 2003:http://www.ha.org.hk/ sars/sars_index_e.html. (accessed on May 1, 2004).

4 World Health Organization. Case definitions for surveillance of severe acute respiratory syndrome (SARS). May 1, 2003:http:/ /www.who.int/csr/sars/casedefinition. (accessed on May 1, 2004).

5 World Health Organization. Severe acute respiratory syndrome (SARS): multi-country outbreak-update 56. Jun 12, 2003:http://www.who.int/csr/don/2003_06_12/en/. (accessed on May1, 2004).

6 Center for Disease Control and Prevention. What everyone should know about severe acute respiratory syndrome (SARS). Aug 27, 2003:http://www.cdc.gov/ncidod/sars/ basics.htm. (accessed on May 1, 2004).

7 Department of Health, HKSAR. Latest figures on severe acute respiratory syndrome. Oct 15, 2003:http:// www.info.gov.hk/dh/diseases/ap/eng/infected.htm. (accessed May 11, 2004).

8 World Health Organization. Update 27 - One month into the global SARS outbreak: Status of the outbreak and lessons for the immediate future. Apr 11, 2003:http:// www.who.int/csr/sarsarchive/2003_04_11/en/. (accessed on May 1, 2004).

9 Duan SM, Zhao XS, Wen RF, Huang JJ, Pi GH, Zhang SX et al. Stability of SARS coronavirus in human specimens and environment and its sensitivity to heating and UV irradiation. Biomed Environ Sci 2003; 16: 246-255.

10 Sizun J, Yu MW, Talbot PJ. Survival of human coronaviruses 229E and OC43 in suspension and after drying on surfaces: a possible source of hospital-acquired infections. J Hosp Infect 2000; 46: 55-60.

11 Sattar SA, Springthorpe VS, Karim Y, Loro P. Chemical disinfection of non-porous inanimate surfaces 
experimentally contaminated with 4 human pathogenic viruses. Epidemol Infect 1989; 102: 493-505.

12 Center for Disease Control and Prevention. Interim recommendations for cleaning and disinfection of the SARS patient environment. Apr 28, 2003:http://www.cdc.gov/ ncidod/sars/cleaningpatientenviro.htm. (accessed May 1, 2004).

13 Centers for Disease Control \& Prevention. Supplement I: Infection Control in Healthcare, Home, and Community Settings. January 8, 2004:www.cdc.gov/ncidod/sars/ guidance/I/index.htm. (accessed May 1, 2004).

14 Rutala WA, APIC Guidelines Committee. APIC guideline of selection and use of disinfectants. Am J Infect Control 1996; 24: 313-342.

15 Chan WM, Yuen K, Fan DS, Lam DS, Chan PK, Sung JJ. Tears and conjunctival scrapings for Coronavirus (SARS$\mathrm{CoV}$ ) in patients with SARS. Br J Ophthalmol 2004; 88: 968-969.

16 Yuen KS, Chan WM, Fan DS, Chong KK, Sung JJ, Lam DS. Ocular screening in severe acute respiratory syndrome. Am J Ophthalmol 2004; 137: 773-774.

17 Seto WH, Tsang D, Yung RW, Ching TY, Ng TK, Ho M et al. Effectiveness of precautions against droplets and contact in prevention of nosocomial transmission of severe acute respiratory syndrome (SARS). Lancet 2003; 361: 15191520.

18 Loon SC, Teoh SC, Oon LL, Se-Thoe SY, Ling AE, Leo YS et al. The severe acute respiratory syndrome coronavirus in tears. Br J Ophthalmol 2004; 88: 861-863.

19 World Health Organization. Cumulative number of reported probable cases of severe acute respiratory syndrome (SARS). Sept 26, 2003:http://www.who.int/csr/ sars/country/table2003_09_23/en/. (accessed on May 1, 2004).

20 World Health Organization. Alert, verification and public health management of SARS in the post-outbreak period.
Aug 14, 2003:http:/ /www.who.int/csr/sars/postoutbreak/ en/. (accessed May 1, 2004).

21 World Health Organization. New case of laboratoryconfirmed SARS in Guangdong, China - update 5. 31 January 2004:www.who.int/csr/don/2004_01_31/en/. (accessed on May 1, 2004).

22 Guan Y, Peiris JS, Zheng B, Poon LL, Chan KH, Zeng FY et al. Molecular epidemiology of the novel coronavirus that causes severe acute respiratory syndrome. Lancet 2004; 363: 99-104.

23 Guan Y, Zheng BJ, He YQ, Liu XL, Zhuang ZX, Cheung CL et al. Isolation and characterization of viruses related to the SARS coronavirus from animals in southern China. Science 2003; 302: 276-278.

24 Ruan YJ, Wei CL, Ee AL, Vega VB, Thoreau H, Su ST et al. Comparative full-length genome sequence analysis of 14 SARS coronavirus isolates and common mutations associated with putative origins of infection. Lancet 2003; 361: 1779-1785.

25 McCurry J. Japan steps up SARS plans. Lancet 2004; 363: 219.

26 Nicholson KG, Wood JM, Zambon M. Influenza. Lancet 2003; 362: 1733-1745.

27 Chan PK. Outbreak of avian influenza A (H5N1) virus infection in Hong Kong in 1997. Clin Infect Dis 2002; 34(Suppl 2): S58-S64.

28 Ungchusak K, Auewarakul P, Dowell SF, Kitphati R, Auwanit W, Puthavathana P et al. Probable person-toperson transmission of avian influenza A (H5N1). N Engl J Med 2005; 352: 333-340.

29 Katz JM, Lim W, Bridges CB, Rowe T, Hu-primmer J, Lu X et al. Antibody response in individuals infected with avian influenza A (H5N1) viruses and detection of anti-H5 antibody among household and social contacts. J Infect Dis 1999; 180: 1763-1770.

30 Tam JS. Influenza A (H5N1) in Hong Kong: an overview. Vaccine 2002; 20: S77-S81. 